\title{
Application of Fourier-Pade Approximation in Analysis of Holographic Diffraction Gratings
}

\author{
D. Sevic*, S. Savic-Sevic, D. Pantelic and B. Marinkovic \\ Institute of Physics, University of Belgrade, Zemun Pregrevica 118, P.O. Box 68, 11080 Belgrade, Serbia \\ (Received February 24, 2012; in final form July 9, 2013) \\ The diffraction gratings, fabricated in our laboratory, were presented and analyzed by atomic force microscopy \\ in earlier publications. In this paper we apply the Fourier-Pade approximation on our data previously analyzed \\ by fast Fourier transform, proving advantages of using the Fourier-Pade approximation for spectral analysis of \\ structural properties of some optical materials.
}

DOI: 10.12693/APhysPolA.124.619

PACS: $02.30 . \mathrm{Mv}, 02.30 . \mathrm{Nw}, 42.40 . \mathrm{Eq}$

\section{Introduction}

Fast Fourier transform (FFT) is widespread tool in spectral analysis, primarily because of its computational efficiency and a steady convergence as a function of increasing the sequence length. In this paper a method based on the Fourier transform and Pade approximants $[1,2]$ is investigated for analysis of holographic diffraction gratings. Pade approximants are often used to accelerate the convergence of a slowly converging sequence. Namely, for a power series

$$
F_{n}(z)=\sum_{n=0}^{N} c_{k} z^{k}
$$

of order $N$ in the variable $z$ (real or complex) with coefficients $c_{k}$, the Padé approximant is a rational fraction $P_{m}(z) / Q_{n}(z)$, which approximates the fully converged values of the function $F_{n}(z)$. If we let $z=\mathrm{e}^{-\mathrm{j} k n}$, then Eq. (1) calculates discrete Fourier transform (DFT), so the Pade method could be used to improve spectral resolution of DFT. The method is usually called the FourierPade (FP) approximation.

In this analysis we applied Fourier-Pade approximation on data acquired by atomic force microscopy (AFM) scans of diffraction grating investigated in earlier papers $[3,4]$. We recently used FP for analysis of holographic photonic crystal structures [5]. All obtained results proved advantages of using FP for spectral analysis of structural properties of optical materials instead of FFT. Our calculation of FP is efficiently implemented as a $\mathrm{C}$ code. The computer running time of calculating FP is only slightly longer than by evaluating the FFT.

\section{Analyzed samples}

The diffraction gratings fabricated in our laboratory were analyzed by AFM. Figure 1 shows a zoomed part of sinusoidal shaped grating recorded in dextran sensitized with ammonium dichromate (DCD) [3] (left side of figure). The AFM greyscale image is usually presented in

\footnotetext{
*corresponding author; e-mail: sevic@ipb.ac.rs
}

pseudocolor, where different profile heights are coded as different colors. A corresponding one-dimensional profile is also shown in Fig. 1 (right side).
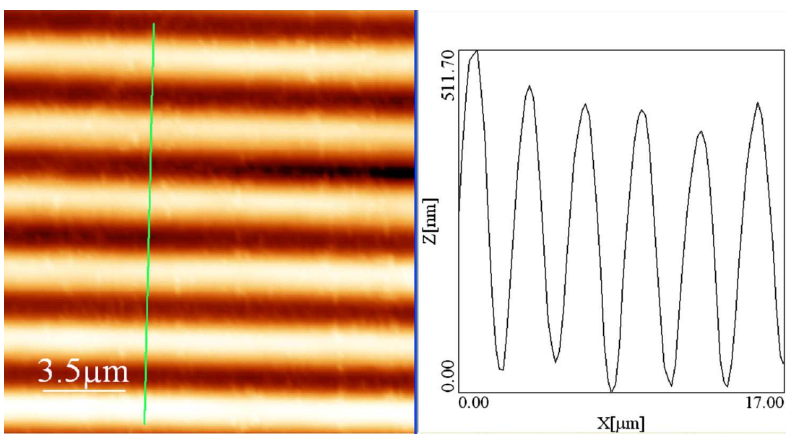

Fig. 1. The zoomed part of AFM image of DCD surface relief diffraction grating with sinusoidal profile (left) and its line profile (right).
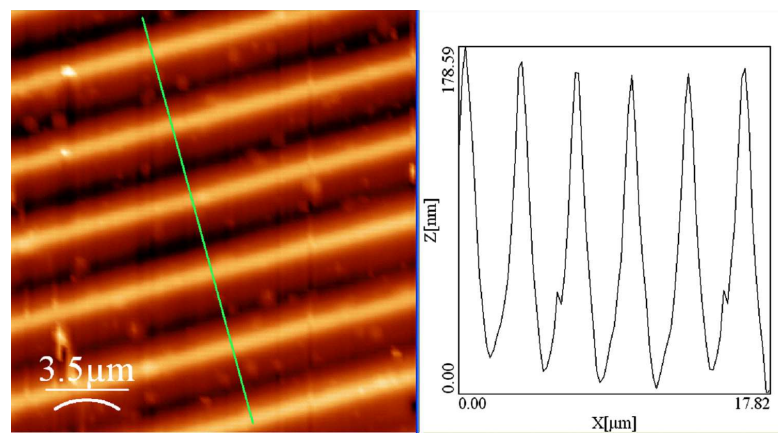

Fig. 2. The zoomed part of AFM image of DCD surfaces relief diffraction grating with non-sinusoidal profile (left) and its line profile (right).

Non-sinusoidal DCD profile grating and its line profile are shown in Fig. 2.

Figure 3 shows a saw-tooth shaped dental polymer (Herculite xrv) grating and its line profile. The grating was made by copying dichromate sensitized pullulan (DCP) grating into dental composite [4]. 

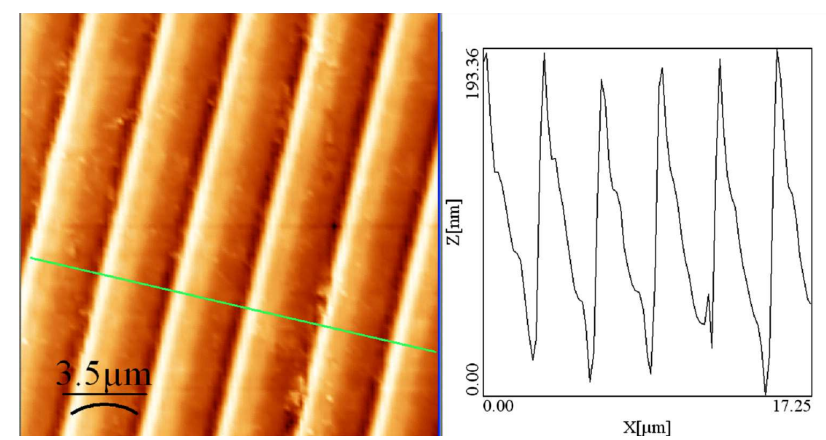

Fig. 3. The zoomed part of AFM image of dental composite (Herculite xrv) diffraction grating (left) and its line profile (right).

\section{Analysis of diffraction grating structures using FP}

Two-dimensional (2D) Pade approximation is not separable, so calculating the $2 \mathrm{D}$ FP is very demanding $[1,2,5]$. Fortunately, diffraction grating structures which are of interest here have directional structure, so we will use the Pade approximation to enhance one-dimensional Fourier analysis of line profiles. The Fourier transform of line profile of the surface relief shown in Fig. 1 is calculated and presented in Fig. 4. Zero frequency (DC)

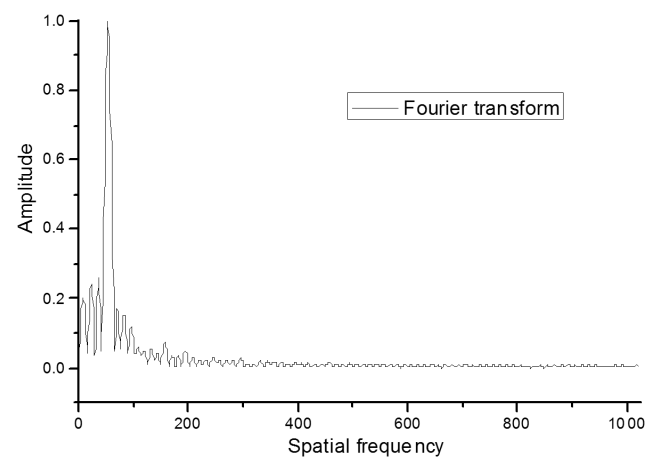

Fig. 4. Fourier transform of line profile of surface relief shown in Fig. 1.

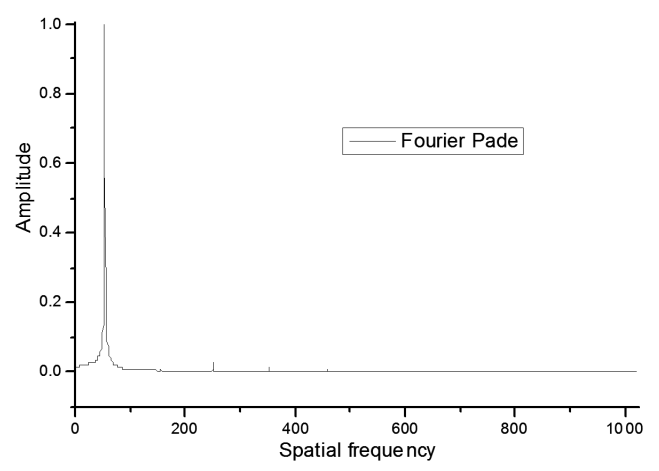

Fig. 5. Fourier-Pade approximation of line profile of surface relief shown in Fig. 1. component is removed by subtracting the average value of the sequence from every point. FP of the same data is calculated and presented in Fig. 5. AFM images analyzed here are $256 \times 256$, so lengths of analyzed line profiles are up to about $\sqrt{2 \times 256}$. To enhance resolution, the Fourier transform of 2048 points is used. The Fourier spectra of real valued data are symmetrical, so only one half of the spectra are presented. Spatial frequency is in the range 0 to $\pi$ and is scaled to 1024 points. Sinusoidal component is easy to discern even in Fig. 4, although there is a lot of "ringing" in FT spectrum. Fundamental sinusoidal component is much more pronounced in FP spectrum. Advantages of using the FP for structural analysis are obvious.

The Fourier transform of line profile of the nonsinusoidal surface relief shown in Fig. 2 is calculated and presented in Fig. 6. DC component is, again, removed. FP of the same data is calculated and presented in Fig. 7. Fundamental components are possible to notice in Fig. 6, but not clearly. Fundamental components are much clearer on FP spectrum, Fig. 7. Components at about 280, 395, and 420 are visible only on FP spectrum. In this case, FP provides much better spectral analysis. However, it should be noted that aforesaid components could be numerical effects caused by the fact that line profile is not orthogonal to the horizontal axis of AFM image. Then,

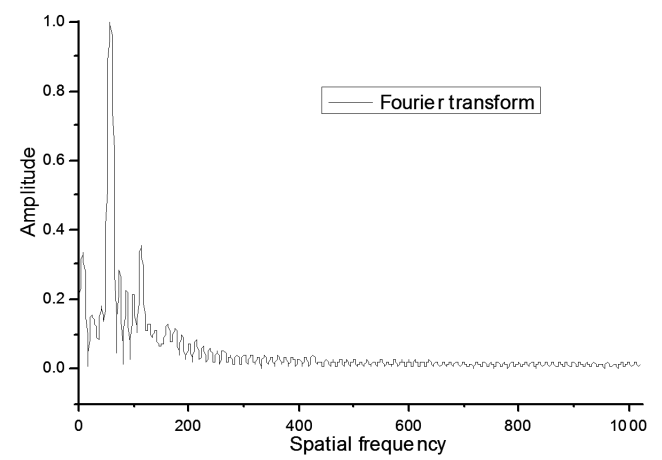

Fig. 6. Fourier transform of line profile of nonsinusoidal surface relief shown in Fig. 2.

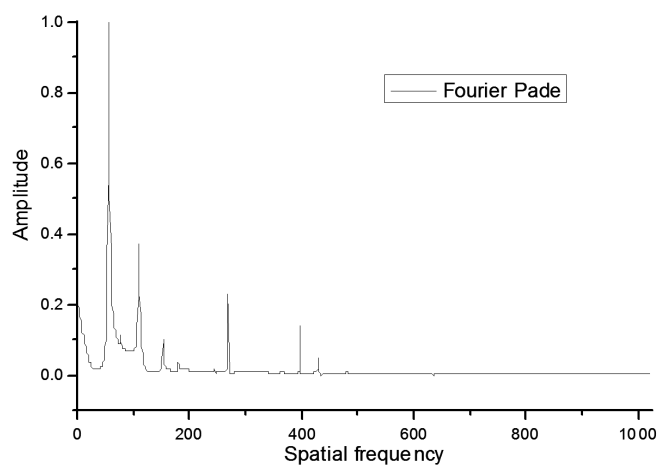

Fig. 7. Fourier-Pade approximation of line profile of nonsinusoidal surface relief shown in Fig. 2. 
the values of the line profile are interpolated through orthogonally sampled points of AFM image.

The Fourier transform of line profile of the saw-tooth shaped surface relief shown in Fig. 3 is calculated and presented in Fig. 8. DC component is, again, removed. FP of the same data is calculated and presented in Fig. 9. Saw-tooth sequence has plenty of components which are not quite possible to identify in Fig. 8. Components are easier to identify on FP spectrum, Fig. 9. FP analysis provides enhanced insight into spatial frequency characteristics of diffraction grating structures.

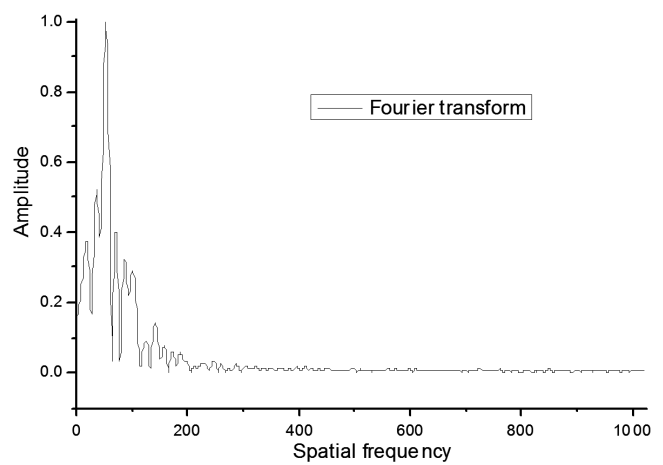

Fig. 8. Fourier transform of line profile of saw-tooth shaped surface relief shown in Fig. 3.

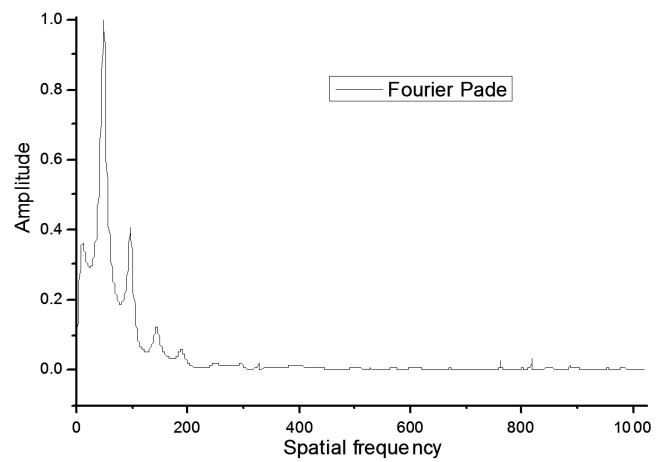

Fig. 9. Fourier-Pade approximation of line profile of saw-tooth shaped surface relief shown in Fig. 3.

It should be commented here that although with such superior performance, as presented in this study, FP is not widely used as FFT. There are several reasons for this. First of all, as already mentioned, the FFT is the most popular tool for spectral analysis because of its simplicity of using and availability. The Fourier-Pade approximation is not so simple to use, moreover, it is not available as application software. Computational complexity of length- $N$ FFT is of order of $(N \log N)$ arithmetic operations, while FP requires several orders of magnitude larger number of operations, depending on the rate of its convergence for the specific case. However, for relatively short one-dimensional data sequences analyzed in this paper, the computer running time of calculating FP is just slightly longer than when computing the FFT.

\section{Conclusion}

In this paper a method based on the Fourier transform and Pade approximants is investigated for structural analysis of diffraction gratings fabricated in our laboratory. Compared to the conventional use of the Fourier transform, structural analysis of fabricated samples is enhanced by better spatial frequency resolution provided by the Fourier-Pade approximation. Spectral components which are just discernible in the Fourier spectrum are more pronounced when using the Fourier-Pade approximation. We implemented computing of the Fourier-Pade approximation as a $\mathrm{C}$ code.

\section{Acknowledgments}

The work has been done within the projects OI 171020 , OI 171038 and III45016 financed by the Ministry of Education and Science, Republic of Serbia.

\section{References}

[1] M.F. Calaghan, D.J. Larkman, J.V. Hajnal, Magn. Res. Med. 54, 1490 (2005).

[2] Dz. Belkic, Nucl. Instrum. Methods Phys. Res. A 471, 165 (2001).

[3] S. Savic-Sevic, D. Pantelic, Opt. Mater. 30, 1205 (2008).

[4] S. Savic-Sevic, D. Pantelic, Opt. Expr. 13, 2747 (2005).

[5] D. Sevic, S. Savic-Sevic, D. Pantelic, B. Jelenkovic, B.P. Marinkovic, Acta Phys. Pol. A 116, 647 (2009) 